\title{
My Hips Hurt: An Unusual Presentation of Bilateral Groin Pain in an Adolescent Boy
}

Herman H.Y. Tam ${ }^{\circledR}$, MBBS, MSc, Division of Rheumatology, British Columbia Children's Hospital, Vancouver, British Columbia; Jennifer Stimec, MD, Department of Diagnostic Imaging, The Hospital for Sick Children, Toronto, Ontario; Shirley M.L. Tse, MD, Division of Rheumatology, The Hospital for Sick Children, Toronto, Ontario, Canada. Address correspondence to Dr. H. Tam, Pediatric Rheumatologist, Division of Rheumatology, British Columbia Children's Hospital, 4480 Oak Street, Vancouver, BC V6H 3V4, Canada. Email: tamherman@gmail.com. The authors declare no conflicts of interest relevant to this article. Ethics approval was not required for deidentified case report as per institutional policy. Consent was obtained from the patient.

Van Neck-Odelberg disease (VND) is a rare benign pediatric skeletal abnormality characterized by hyperostosis of the ischiopubic synchondrosis (IPS) and clinical symptoms. ${ }^{1,2,3}$ IPS is a strip of cartilaginous tissue between the superomedial pubic and posterolateral ischial ossification centers, which develops into the inferior pubic rami and ischial tuberosity, respectively.

A 12-year-old boy presented to the pediatric rheumatology clinic with 18 months of mild intermittent bilateral groin pain and a limp. Physical examination demonstrated normal hip exam with mild pain at end range of motion (left greater than right). Radiograph showed expansion of IPS bilaterally with normal hips (Figure 1). Magnetic resonance imaging demonstrated left fusiform IPS expansion with increased signal intensity in the bone and adjacent soft tissue; and absence of synovitis, soft tissue mass, or collections (Figure 2A,B). He was diagnosed with VND correlating with radiographic changes. His symptoms completely resolved with conservative management.

IPS usually closes asymptomatically followed by bony fusion during late childhood. However, some children may develop symptoms of groin pain and restricted hip movements, as well as a limp, leading to VND. Radiographic changes include radiolucencies, bony enlargement, osteopenia, and calcifications. Most importantly, the absence of synovitis, soft tissue mass, or collections is helpful in excluding other differentials including transient synovitis, fracture, infections, and neoplasm. Increased clinical and radiographic recognition can avoid unnecessary diagnostics and treatments that are frequently performed. ${ }^{4}$ Management is supportive as symptoms resolve with skeletal maturity. In summary, VND should be considered in children presenting with groin pain and distinctive radiographic findings.

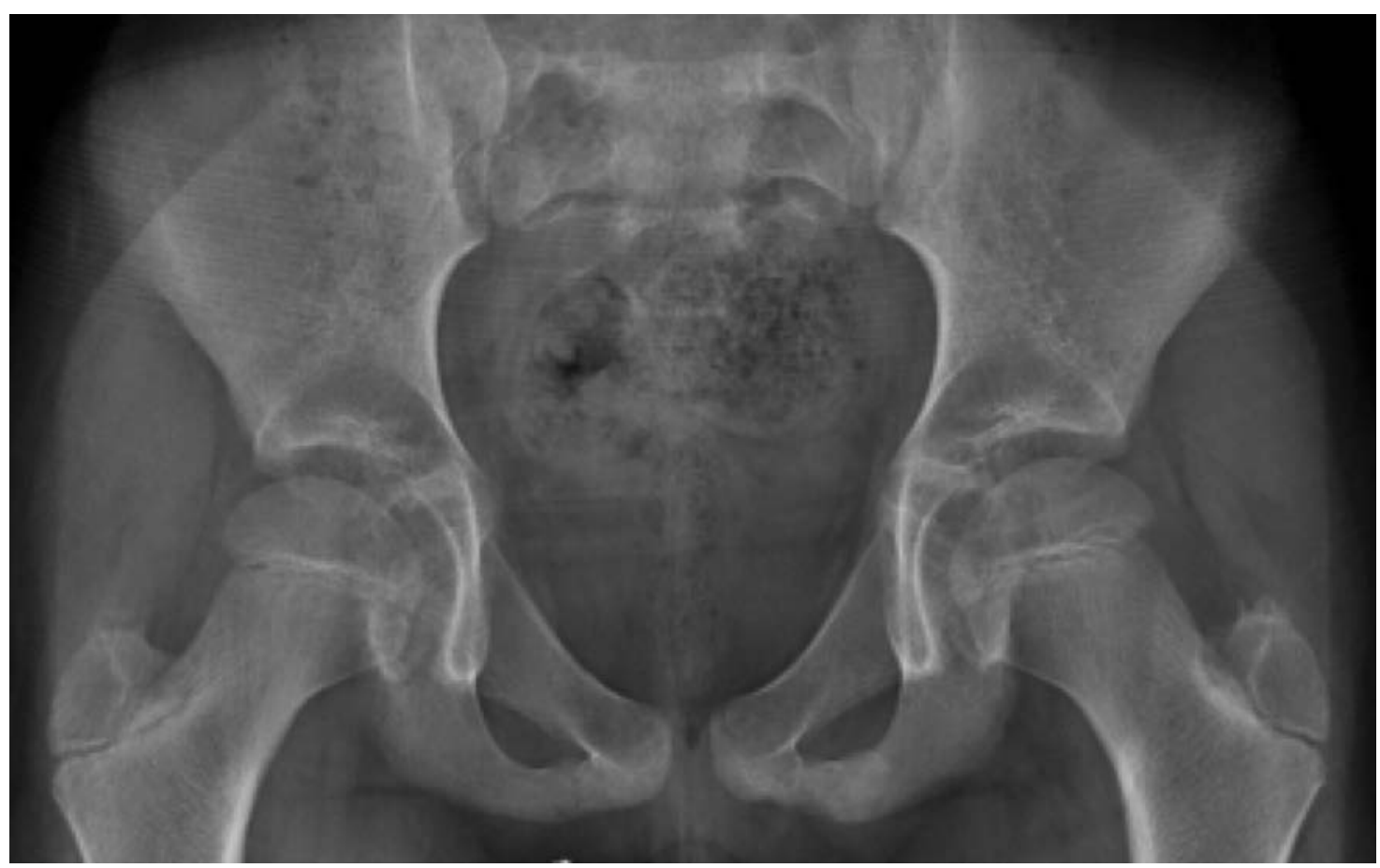

Figure 1. Radiograph demonstrates expansion of IPS bilaterally (left > right) with normal hips. IPS: ischiopubic synchondrosis. 


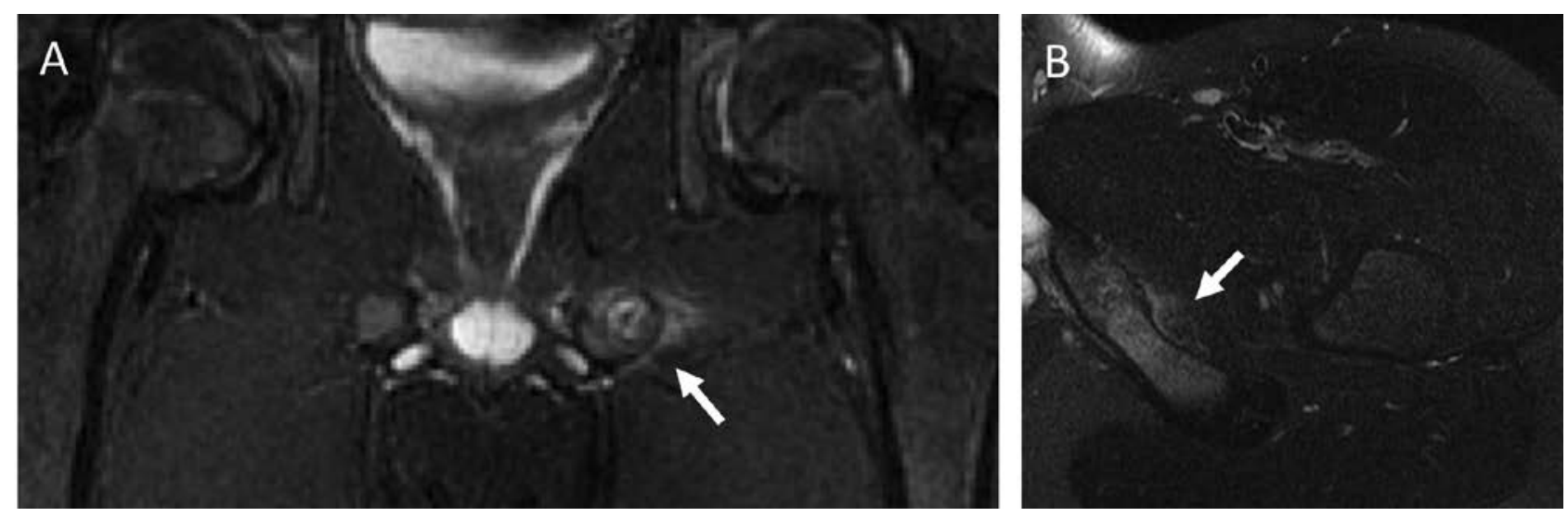

Figure 2. MRI (A) coronal STIR and (B) axial T2-FS demonstrate fusiform expansion at the left IPS (white arrows) with increased signal intensity in the bone and adjacent soft tissues. Note the absence of a soft tissue mass or collection. The right IPS appears normal. MRI: magnetic resonance imaging. IPS: ischiopubic synchondrosis; STIR: short-tau inversion recovery; T2-FS: fat-saturated T2-weighted imaging.

\section{REFERENCES}

1. Odelberg A. Some cases of destruction in the ischium of doubtful etiology. Acta Chir Scand 1923;56:273-84.

2. Van Neck M. [Osteochondritis of the pubis.] [Article in French] Arch Franco-Belges Chir 1924;27:238-41.

3. Wait A, Gaskill T, Sarwar Z, Busch M. Van neck disease: osteochondrosis of the ischiopubic synchondrosis. J Pediatr Orthop 2011;31:520-4.
4. Schneider KN, Lampe LP, Gosheger G, Theil C, Masthoff M, Rödl R, et al. Invasive diagnostic and therapeutic measures are unnecessary in patients with symptomatic van Neck-Odelberg disease (ischiopubic synchondrosis): a retrospective single-center study of 21 patients with median follow-up of 5 years. Acta Orthop 2021;92:347-51. 